\title{
Formación de equipos de alto rendimiento con estudiantes de ingeniería, a través de cohesión y madurez grupal y su aplicación en un proyecto específico
}

\section{Formation of high performance teams with engineering students, through group cohesion and maturity and their application in a specific project}

TLAPALE-HERNÁNDEZ, Prudencio †*, PÉREZ-RAMOS, María Gabriela, MACUIL-CARMONA, Carolina y MERINO-MUÑOZ, Víctor Hugo

Universidad Tecnológica de Huejotzingo, Puebla, México

ID $1^{\text {er }}$ Autor: Prudencio, Tlapale-Hernández / ORC ID: 0000-0003-3309-5977, CVU CONACYT ID: 813440

ID $1^{\text {er }}$ Coautor: María Gabriela, Pérez-Ramos / ORC ID: 0000-0001-9016-0099, Researcher ID Thomson: Q-6647-2018, CVU CONACYT ID: 458844

ID $2^{\text {do }}$ Coautor: Carolina Macuil-Carmona / ORC ID: 0000-0001-7218-6850, CVU CONACYT ID: 909782

ID $3^{\text {er }}$ Coautor: Víctor Hugo, Merino-Muñoz / ORC ID: 0000-0002-1335-6458, Researcher ID Thomson: Q-6683-2018, CVU CONACYT ID: 940924

\section{Resumen}

Este trabajo es resultado de interpretar y aplicar teorías conductuales de autores como Skinner, Maslow, Herzberg y Mc Gregor, con estudiantes de ingeniería, para fortalecer destrezas y habilidades interpersonales con el objetivo de integrarlos en EAR (equipos de alto rendimiento), evaluándolas mediante la implementación en un proyecto de disminución de pago por consumo de energía eléctrica en sus hogares. El estudio es de tipo experimental descriptivo, desarrollado con 68 estudiantes de IPOI (Ingeniería en Procesos y Operaciones Industriales) de la UTH (Universidad Tecnológica de Huejotzingo), midiéndolo con respuestas a cuestionarios propuestos en la literatura sobre la cohesión y madurez de los EAR y estableciendo relaciones con el resultado del proyecto. La integración, seguimiento y evaluación, son parte fundamental del método, el cual a través de los resultados del grado de cohesión (54.3\%) y madurez $(50.46 \%)$, adquiere importancia en el análisis de correlación con valores de 0.7803 y 0.8197 , respectivamente, en el ahorro de energía. El proyecto contribuye a mejorar el esquema didáctico de integración de alumnos en EAR a través de la actitud, satisfacción de necesidades, y predisposición para superar expectativas; observando cómo se agrupan, se descartan, se reagrupan y se convierten en seguidores.

Equipo de Alto Rendimiento, Teorías conductuales, Método

\begin{abstract}
The following paper gathers the results of applying and interpreting behavioral theories from authors such as Skinner, Maslow, Herzberg and Mc Gregor, among engineering students, to further strengthen their interpersonal skills and abilities aiming at their later incorporation into EAR (high performance teams), evaluating them through their implementation in electric energy efficiency projects at home. The study is descriptive and experimental, which was developed with 68 IPOI students (Processes and Industrial Operations Engineering) from UTH (Universidad Tecnológica de Huejotzingo), measuring it through tests' answers given in the same literature about EAR maturity and union, thus stablishing a relation with the project result. Alliance, tracking and evaluating are an essential part of the method, which through the union $(54.3 \%)$ and maturity $(50.46 \%)$ grade results has acquired relevance in the correlation analysis with 0.7803 and 0.8197 values respectively in the electric energy saving. The project contributes to improve the didactic overview about student's integration in EAR through attitude, satisfaction of needs and willingness to exceed expectations, observing how they are grouped, discarded, regrouped and turned into followers.
\end{abstract}

High performance team, Behavioral theories, Method

Citación: TLAPALE-HERNÁNDEZ, Prudencio, PÉREZ-RAMOS, María Gabriela, MACUIL-CARMONA, Carolina y MERINO-MUÑOZ, Víctor Hugo. Formación de equipos de alto rendimiento con estudiantes de ingeniería, a través de cohesión y madurez grupal y su aplicación en un proyecto específico. Revista de Ciencias de la Educación. 2019. 3-7: 11-20

\footnotetext{
* Correspondencia del Autor (correo electrónico: prudencio.tlapale@uth.edu.mx)

$\dagger$ Investigador contribuyendo como primer autor.
} 


\section{Introducción}

La educación superior, desde nuestro punto de vista, debe promover la adaptación de los estudiantes al proceso enseñanza aprendizaje a través de técnicas pedagógicas y didácticas que los doten de una autonomía orientada e incorporada a conseguir las competencias profesionales, enfatizando en los temas de trabajo en equipo y equipos de alto rendimiento, pues su empleabilidad dependerá menos de lo que conozcan y más de su capacidad de aprender, adaptarse y ejecutar.

A las universidades nos alcanzaron las generaciones de jóvenes millennials y dentro de poco los jóvenes alpha superarán nuestra expectativa docente, ambas cuentan con una capacidad extraordinaria de recursos tecnológicos que les ayudan en su experiencia didáctica lo que les ha desarrollado una gran habilidad tecnológica, haciendo de estas personas altamente competitivas en recursos digitales, sin embargo, observamos que esa velocidad a la que se desarrollan es la misma a la que pierden el interés por los procesos, haciendo entonces, que el establecimiento de metas y objetivos al interior de las universidades y empresas sean claros y dinámicos para mejorar su estructura y conseguir los resultados esperados.

De acuerdo con el Instituto mexicano de competitividad (IMCO, 2017), las competencias valoradas por el mercado laboral demandarán para el 2020: 1) solución de problemas complejos, 2) Pensamiento crítico, 3) Creatividad, 4) Manejo de personas, 5) Coordinación con otros, 6) Inteligencia emocional, 7) Toma de decisiones, 8) Orientación de servicio, 9) Negociación y 10) Flexibilidad cognitiva. De este análisis los puntos 4,5, y 6 tienen una estrecha relación con el tema en comento y una importancia relevante al impartirse en el sistema de formación tecnológica. Para este estudio utilizamos algunas teorías para conseguir la integración de los estudiantes, el seguimiento de sus actividades y la evaluación de las mismas como participantes en equipos de alto rendimiento, incorporándolas a su proceso formativo con una visión clara y objetiva, considerando el ambiente universitario, la edad de los participantes, el tamaño de los grupos, los materiales didácticos y el tiempo asignado por sesión; sin olvidar la formación profesional del docente.
Rompiendo el encapsulado del aprendizaje estático y convirtiéndolo en dinámico, este cambio beneficia y modifica la forma de trabajar del docente, así como la de aprender del estudiante. Manifestando que los conceptos de enseñar y aprender están directamente vinculados con el proceso formativo de los estudiantes como lo comenta López (2005) al mencionar que "enseñar y aprender son dos caras de una misma moneda ya que una va unidad indefectiblemente a la otra".

A partir del modelo de educación basado en competencias para la educación superior, se requiere formar profesionistas que sean dinámicos y flexibles, para desempeñarse profesionalmente en el ámbito industrial, por lo tanto, la práctica docente debe generar un ambiente en el que el estudiante aprenda haciendo, razone cada situación aportando conocimientos y experiencias, y plantee soluciones innovadoras y creativas a los problemas que surgen en los procesos productivos.

El estudio se realizó con 68 estudiantes de ingeniería en Procesos y Operaciones Industriales (IPOI) de la Universidad Tecnológica de Huejotzingo (UTH). Para su elaboración se identificaron, se conceptualizaron y se dinamizaron cuatro teorías para observar la actitud, la satisfacción de necesidades, la satisfacción y la predisposición de los estudiantes para superar sus propias expectativas.

El objetivo de esta investigación es aplicar cuatro teorías del estudio del comportamiento humano a través de dinámicas aplicadas a la población objeto de estudio para explorar aspectos de interés sobre la motivación de los estudiantes y conseguir resultados que ayuden a la integración en EAR.

Una característica del estudio es facilitar el proceso enseñanza-aprendizaje demostrando la funcionalidad de los conceptos teóricos con el ejercicio práctico para que las teorías seleccionadas resulten de interés para los estudiantes y les den la oportunidad de mejorar como personas y profesionistas. La elección de los conceptos se hizo de acuerdo con el programa de estudios vigente 2017 , de forma continua sin importar que los temas sean aislados o secuenciales. 
Por lo tanto, se propone el planteamiento lúdico de éstos como una estrategia de apoyo para el docente frente a grupo, para dinamizar el proceso de enseñanza e incrementar el nivel de comprensión de una manera lógica y pertinente en los estudiantes

\section{Problema}

Se ha observado que los estudiantes de la carrera de IPOI, durante todo el proceso formativo y por varias generaciones, han realizan actividades de trabajo en equipo dividiéndose las tareas e integrando los resultados como aportaciones individuales, lo que hace que esta acción se grabe en ellos y posteriormente al ingresar al ámbito laboral no la corrijan o eliminen, ocasionado una inadaptación temprana en el medio empresarial.

\section{Hipótesis}

Con la aplicación de cohesión y madurez grupal se contribuye a mejorar la integración y adaptación de los estudiantes a los equipos de alto rendimiento, como parte de una habilidad suave para su desarrollo, mostrando un universo de posibilidades de aplicación estratégica, práctica y de innovación que favorezcan a la productividad en su entorno profesional.

Para el estudio se construyó el marco teórico resumiendo aspectos de equipos de alto rendimiento y motivación abordando los temas de: 1) Integración de equipos de alto rendimiento, 2) Aplicación de Teorías de la conducta humana y 3) Análisis de resultados obtenidos de las encuestas aplicadas y del desarrollo del proyecto.

\section{Desarrollo}

\section{Marco teórico}

Los aspectos de trabajo en equipo y las teorías psicológicas relacionadas con la conducta del ser humano presentan una reacción hacia el logro de mejores resultados, siendo esta la parte principal del estudio al experimentar y evaluar acciones por repetición, por intuición, por conveniencia, y uno de los más importantes por satisfacción de los integrantes.
Con respecto a los equipos de alto rendimiento, se pretende que los integrantes muestren una atención eficaz para la formación de los equipos de trabajo con la idea de conseguir resultados satisfactorios para las organizaciones. A partir de estos, entendemos que si son satisfactorios y relevantes entonces se consigue la denominación de un equipo de alto rendimiento, pero si son normales o están por debajo de las expectativas son solo equipos de trabajo.

Lipman-Blumen y Leavitt (2000), mencionan que un "equipo de alto rendimiento es una disposición de ánimo general, una actitud compartida, volcada y comprometida a fondo con su tarea, una actitud que puede extenderse a cualquier tipo de equipo". Por lo tanto, ser integrante de un equipo con estas características, no garantiza la satisfacción de sus necesidades, sin embargo, sí brinda la oportunidad de tener experiencias de logro de objetivos en tiempos reducidos.

Los integrantes de estos equipos deben ser conducidos a través de algunos conceptos escritos y algunos otros demostrados como la teoría de Skinner e introducirlos a los aspectos de la teoría de necesidades de Maslow, de los factores higiénicos de Herzberg y la teoría "X" y "Y" de McGregor, con la finalidad de generarles elementos suficientes que coadyuven a su integración en los equipos de alto rendimiento, tarea difícil pero no imposible pues como menciona Skinner (1994) "un solo caso basta para quitar la razón a los que piensan que es algo irrealizable".

Así el condicionamiento operante de Skinner, descrito por Smith (1994) menciona que las respuestas, el refuerzo a las acciones y el manejo de los estímulos son una parte de la conducta de las personas que nos llevan al manejo práctico, técnico y concreto de las actividades, por lo que, en este sentido, se pretende que los estudiantes adquieran cierto conocimiento y el desarrollo de habilidades para dirigir el esfuerzo de sus subordinados.

Este enfoque, nos conduce a establecer una analogía con el principio físico de "a toda acción corresponde una reacción de igual magnitud y dirección, pero en sentido contrario", el cual trasladado al principio en comento, nos conduce a esperar en principio, una reacción negativa del subordinado. 
Sin embargo, si se aplica un refuerzo aceptable y asimilable por el subordinado este se convierte en una reacción positiva, ambas reacciones expresan una razón actitudinal con la que los colaboradores se comportan como deberían y no como esperaríamos que lo hicieran bajo nuestro marco referencial, modificando de esta manera la previsión de la conducta.

Una vez que el integrante del equipo se ha convencido de su incorporación, a través de la modificación conductual, inicia un proceso de motivación para mantenerse e incluso ascender dentro de las organizaciones mediante la obtención de mejores resultados de las actividades emprendidas.

Por lo tanto, es preciso destacar el término motivación (RAE, $23^{\mathrm{a}}$ edición), como el "conjunto de factores internos o externos que determinan en parte las acciones de una persona", una definición adicional con carácter técnico es la enunciada por Santrock (2002) en su libro tecnología de la educación mencionando que es "el conjunto de razones por las que las personas se comportan de las formas en que lo hacen. El comportamiento motivado es vigoroso, dirigido y sostenido". La cual tiene una relación estrecha con el comportamiento de las personas que toman la decisión de hacer trascender sus acciones positivas.

En el mismo sentido, Medina (2008) comentan "que la motivación se refiere al impulso y esfuerzo por satisfacer un objetivo o expectativa", introduciéndonos bajo estos conceptos a la teoría de necesidades de Maslow (1991), las cuales y desde nuestro punto de vista, no tiene ninguna relación con la motivación, sin embargo, son utilizadas como un medio general en la estructura de la sociedad para conseguir lo que una persona necesita para satisfacer sus expectativas profesionales y personales.

Para satisfacerlas es necesario que cada integrante sea motivado de manera intrínseca o extrínseca, desarrollando y poniendo en juego todo su potencial, su creatividad y su talento, para alcanzar sus objetivos y los del equipo de alto rendimiento. Por lo tanto, la motivación, es un impulso al logro del resultado para conseguir la satisfacción que cubre las necesidades de la pirámide de Maslow (1991), sin omitir desde luego, que el integrante podría conseguir una alta satisfacción, pero al mismo tiempo un bajo nivel de motivación.
En el sentido de los factores higiénicos de Herzberg (1959), menciona en su teoría que la satisfacción laboral dependerá del grado de satisfacción de dos factores, el primero: relacionado con el ambiente que rodea a las personas, directamente administrado y decidido por las organizaciones; y el segundo: relacionado directamente con la satisfacción de la persona. El primer factor es susceptible de controlarse o incluso eliminarse al considerase efímeros, sin embargo, para el segundo factor es preciso enfatizar en la forma de sentirse bien como individuo haciendo las actividades que nos dejan una satisfacción personal, que nos conducen a la satisfacción de las necesidades y por tanto a conseguir resultados satisfactorios y al cumplimento de objetivos.

Por su parte Mc Gregor (1986), desarrolló su teoría de $\mathrm{X}$ y $\mathrm{Y}$ a partir de dos hipótesis contrarias sobre la motivación y predisposición de las personas para realizar tareas; refiriendo para el factor humano que la teoría $\mathrm{X}$ es un proceso de dirigir sus esfuerzos, motivarles, controlar sus acciones y modificar sus conductas para ajustarlas a las necesidades del entorno laboral; en tanto que la teoría Y para el mismo factor, menciona que las personas no son pasivas o resistentes por naturaleza a los cambios, son entes motivados, con deseos de potencializar su desarrollo, de asumir responsabilidades y con disposición para dirigir la conducta hacia los fines organizativos.

\section{Metodología}

De acuerdo a las características del proyecto la metodología empleada en el estudio es de tipo experimental-descriptiva, ya que se busca facilitar la integración de los estudiantes en EAR mejorando su actitud y motivación en el desempeño de actividades, buscando resultados que evidencien la utilización y el aprovechamiento de las teorías por cada estudiante, agrupándolos y analizándolos uno a uno para determinar el nivel de mejora y logro.

\section{Sujetos de studio}

Se tomó como conjunto de estudio un total de 68 estudiantes en la UTH, del décimo cuatrimestre de IPOI, para trabajar una sesión por semana de dos horas cada vez, en horarios vespertinos que oscilaron entre las 14:00 y las 16:00 horas, con una duración total de 30 horas. 


\section{Instrumentos}

Para determinar el grado de cohesión alcanzado por los integrantes de los equipos se realizó una adaptación del instrumento "Group Environment Questionnaire (GEQ)" utilizados para evaluar esta cualidad en equipos deportivos por Prokešová (2011). El cuestionario tiene cinco opciones de respuesta: a) en completo desacuerdo, b) en desacuerdo, c) ni de acuerdo ni en desacuerdo, d) de acuerdo y e) completamente de acuerdo. Para la evaluación cuantitativa se utilizó una escala del uno al cinco y se analizó el entorno total de las respuestas.

En cuanto al grado de madurez de los equipos, se utilizó el instrumento "Team Maturity Questionnaire Electronically" desarrollado por Tuckman y tomado de educationdocbox.com, (2019).

\section{Procesamiento de la información}

Se utilizó el programa informático de Excel, para capturar las 1224 respuestas del cuestionario adaptado (GEQ) y 2176 del cuestionario de madurez, contestadas por los 68 estudiantes que participaron en el proyecto. Las cuales se agruparon por similitud de características para asignarles el nombre del dominio y atributos (ver tabla 1) y realizar el estudio de correlación.

\begin{tabular}{|c|c|c|}
\hline $\mathrm{N}^{\circ}$ & Dominio & Atributo \\
\hline 1 & Cohesión grupal & $\begin{array}{ll}\text { Tendencia } & \text { a } \\
\text { permanecer unidos }\end{array}$ \\
\hline 2 & Madurez grupal & $\begin{array}{l}\text { Identificar la etapa de } \\
\text { trabajo }\end{array}$ \\
\hline 3 & $\begin{array}{l}\text { Correlación entre variables, } \\
\text { cohesión y madurez VS } \\
\text { ahorro en el consumo de } \\
\text { energía eléctrica }\end{array}$ & $\begin{array}{llr}\text { Utilización } & \text { de } & \text { los } \\
\text { instrumentos } & \text { y manejo } \\
\text { estadístico } & \text { de } & \text { la } \\
\text { información } & & \\
\end{array}$ \\
\hline 4 & $\begin{array}{l}\text { Oportunidad para el estudio } \\
\text { de las variables }\end{array}$ & 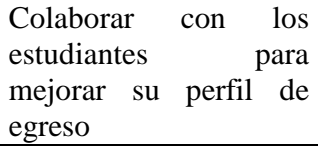 \\
\hline
\end{tabular}

Tabla 1 Definición de variables

Fuente: Elaboración Propia IPOI-UTH

\section{Variables de studio}

Las variables definidas para este estudio son:

a) Cohesión y madurez grupal para integrar a los estudiantes de ingeniería en equipos de AR b) Disminución del consumo de energía eléctrica, al menos en un $10 \%$, en las casas de los participantes, como una consecuencia de alcanzar la integración en equipos de AR

\section{Fases del método}

\section{a) Integración}

Se toma como antecedente el que los estudiantes, desde siempre y durante los cuatrimestres anteriores de su formación profesional han desarrollado actividades en equipo, sin embargo, es en esta última etapa cuando se hace necesario eliminar formas de agrupación ortodoxas y aplicar las teorías desarrolladas por investigadores en este campo, esta es la razón del porque nuestra propuesta de integración se hizo siguiendo un proceso normal y otro dirigido de tal manera que los participantes no se sintieran presionados y molestos por el proceso. Así en el esquema de la figura 1 se representa el proceso de esta fase.

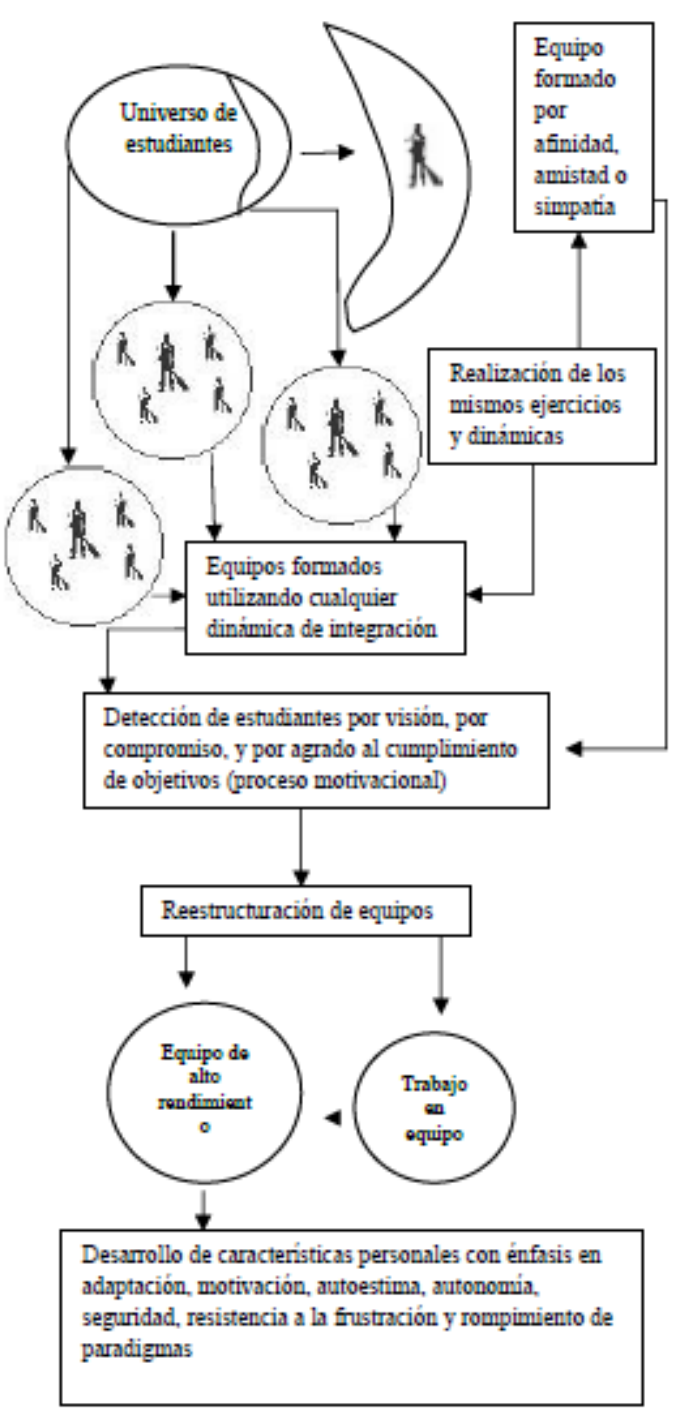

Figura 1 Representación de la integración de los EAR Fuente Propia IPOI-UTH

TLAPALE-HERNÁNDEZ, Prudencio, PÉREZ-RAMOS, María Gabriela, MACUIL-CARMONA, Carolina y MERINO-MUÑOZ, Víctor Hugo. Formación de equipos de alto rendimiento con estudiantes de ingeniería, a través de cohesión y madurez grupal y su aplicación en un proyecto específico. Revista de Ciencias de la Educación. 2019 
b)

\section{Desarrollo del método aplicando las teorías}

Se inició trabajando con el condicionamiento operante, dirigido al aprendizaje de la integración en el que se explica la conducta asociada a la voluntad del cuerpo y como se relaciona con el trabajo. Experimentalmente se plantea que para conseguir lo que el estudiante anhela es preciso trabajar de una forma similar a lo representado en la figura 2.

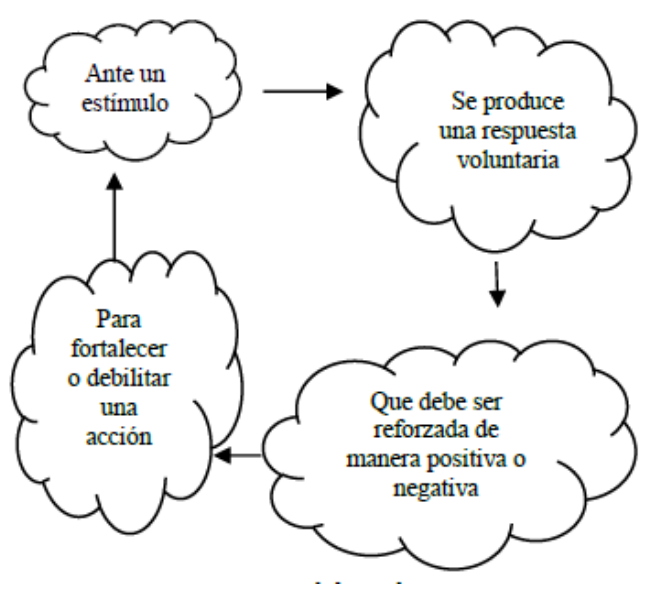

Figura 2 Representación del condicionamiento operante Fuente Propia IPOI-UTH

En el inicio asociamos el estímulo como parte de una necesidad por hacer o poseer algo y que para conseguirlo es necesario admitir un nivel de motivación y enfocarnos a los niveles de la pirámide de Maslow. A continuación, se trabajó con la teoría de Maslow, descartando aquellos aspectos que el estudiante tiene cubiertos como integrante de una familia y enfocándose a aquellos, mostrados en la figura 3 , de su interés como son la necesidad de afiliación y la de reconocimiento, la de autorrealización está fuera de su objetivo y reconocen que solo a través del tiempo y de los resultados podrán conseguirla.

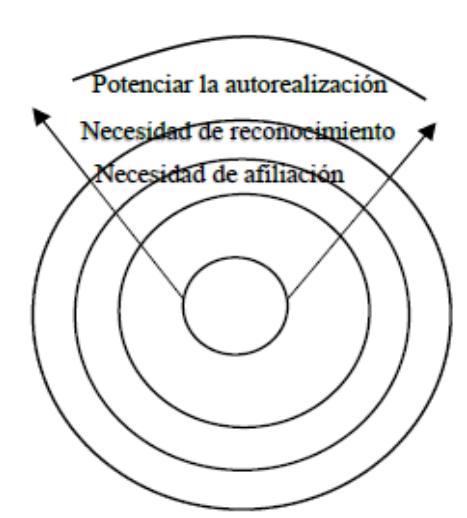

Figura 3 Necesidades de interés a desarrollar Fuente Propia IPOI-UTH
Se tomó como base para el proceso la adaptación de la dinámica de la NASA (Pere, 2014) considerando 16 cosas tangibles y 16 intangibles, inicia el ejercicio descartando 4 de estas, en un segundo ejercicio se prescinde de 4 más y finalmente se deja en la lista del estudiante solo 4 cosas, las cuales potenciará para conseguir su desarrollo profesional.

Pasando a la teoría X y Y de Mc Gregor, se enfatizó en tres principios: 1) generar confianza con los integrantes de los equipos, permitiéndoles incluso pequeños errores susceptibles de corregirse de forma inmediata; 2) atender las relaciones humanas, para aumentar la satisfacción y el compromiso, por las actividades, para reducir su resistencia al cambio y aceptar la autoridad formal, haciendo que descubran con entusiasmo y satisfacción lo que les permitió mejorar en la toma de decisiones y; 3) estrechar las relaciones sociales, con el sentido de que las actividades dependen de la atracción personal derivada de la amistad generada entre los integrantes para finalmente terminar en una relación de trabajo interactiva.

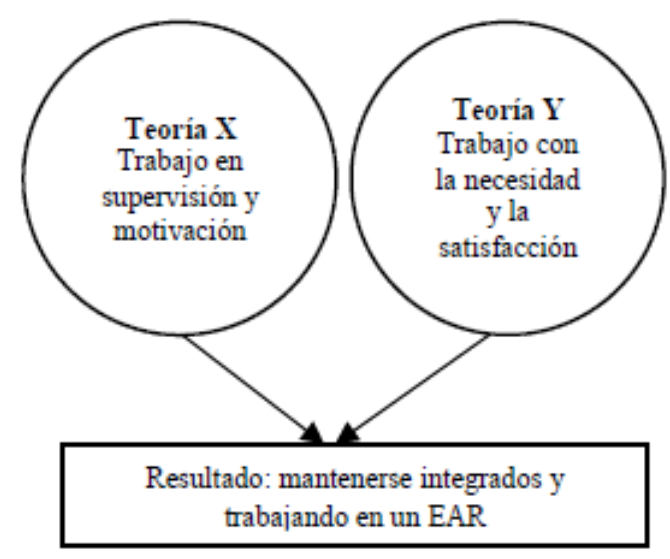

Figura 4 Asociación de teorías X y Y, a la integración de EAR

Fuente Propia IPOI-UTH

La figura 4, resume el cambio de las actividades de supervisión de tareas y motivación de los integrantes en lugar de la imposición y la amenaza que prevalece en la teoría $\mathrm{X}$; en cuanto a la teoría $\mathrm{Y}$, se fortalece la necesidad de trabajar y se refuerza la satisfacción de la actividad de tal manera que los integrantes del EAR encontraran formas de conseguir los mejores resultados. 
Con respecto a los factores higiénicos de Herzberg, se trabajó primero con la parte extrínseca o higiénica para que los integrantes de los EAR se familiaricen con la insatisfacción, buscando minimizarla, ya que esta se localiza en el ambiente que les rodea y desafortunadamente abarca las condiciones en que desempeñan sus actividades. Por último, se trabajó con el factor intrínseco, el cual está estrechamente relacionado con la satisfacción en el puesto de trabajo elegido y con la motivación natural que origina desempeñarlo, porque al final lo que tiene un valor es el momento.

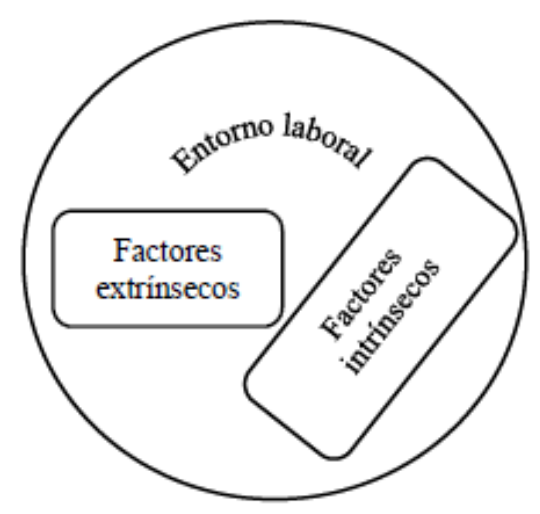

Figura 5 Factores higiénicos en el entorno laboral Fuente Propia IPOI-UTH

Se muestra la representación de los factores extrínsecos minimizados, en la figura 5 , para que el integrante del EAR se percate de la ventaja que tiene, los minimice y cambie hacia los factores extrínsecos buscando tener el control con lo que realiza hasta conseguir su crecimiento y desarrollo personal, su reconocimiento profesional y su autorrealización.

\section{c) Implementación en el Proyecto}

Los estudiantes integraron sus propios EAR para conseguir el objetivo de disminuir en sus hogares el consumo de energía eléctrica y su respectivo pago. Lo hicieron con los integrantes de su familia aplicando lo aprendido en el manejo de las teorías y aplicando conocimientos propios de la ingeniería bajo el siguiente contexto:

- $\quad$ Plantear un objetivo general en el que se mencione realizar actividades encaminadas a conseguir al menos una disminución del $10 \%$ en el pago por el consumo de energía eléctrica contra su último pago.

\section{- Alinear los objetivos específicos con su} aportación para conseguir el objetivo general.

- Desarrollar la justificación del proyecto para empezar a incidir en los integrantes del EAR.

- Definir sus alcances y limitaciones en función de tiempo, conocimientos, economía y actitud de los integrantes.

- Describir las expectativas individuales de cada integrante del EAR y su impacto en el proyecto.

- $\quad$ Elaborar el diagnóstico y su diagrama de causa y efecto, con un enfoque de actitud y conducta de los integrantes, organización de las actividades, consumo excesivo de energía eléctrica, materiales a utilizar y reparaciones eléctricas.

- Elaborar una gráfica de Gantt con horizonte de 15 semanas para verificar el avance del desarrollo y cumplimiento de las actividades propuestas.

- Elaborar un reporte de avances en la semana 5, con énfasis en cohesión del equipo, detección de fallas, control, evidencia del consumo y pago por el servicio. Para la semana 10 , debe contener el grado de madurez alcanzado por los integrantes para conseguir el objetivo y el control del consumo. Para la semana 15, se requiere el control de consumo, la evidencia de pago y sus conclusiones.

\section{Resultados}

\section{Cohesión del equipo}

Se integraron las respuestas en una tabla, con el valor que los estudiantes apreciaron de acuerdo a la evolución del proyecto en el momento solicitado y se muestran en la gráfica 1 . Con esta información se realizó un análisis de frecuencia para datos no agrupados utilizando el promedio por EAR de cada participante, determinado el número de intervalos y su amplitud, ajustándolos a tres categorías para obtener el porcentaje de cohesión. 


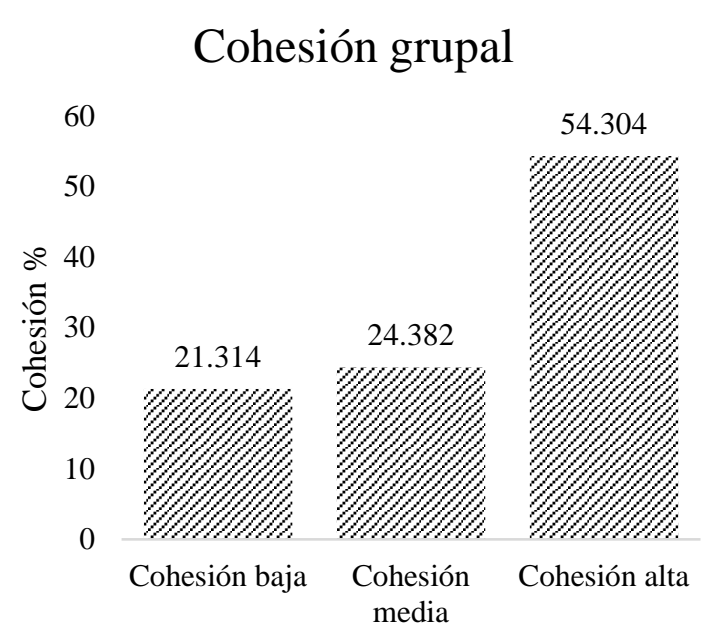

Gráfica 1 Cohesión conseguida por los integrantes de los EAR

Fuente Propia IPOI-UTH

El resultado de cohesión adquirido hasta la semana cinco es aceptable con más del $50 \%$, lo que motiva a los estudiantes a seguir trabajando en el proyecto, aplicando conocimiento y enriqueciéndolo con la aportación de los integrantes. Se resalta que el proceso de integración no se detiene a hacer el análisis de las causas de los porcentajes bajos, pues no es este el objetivo; por lo tanto, el proyecto sigue su marcha enfocado a los equipos que presentan cohesión alta y media, para los de cohesión baja solo se refuerza en la etapa de madurez.

\section{Grado de madurez}

Se realizó el análisis de datos aportados por los responsables de equipos consiguiendo los resultados que se presentan en la gráfica 2, para cada etapa de la integración de lo EAR

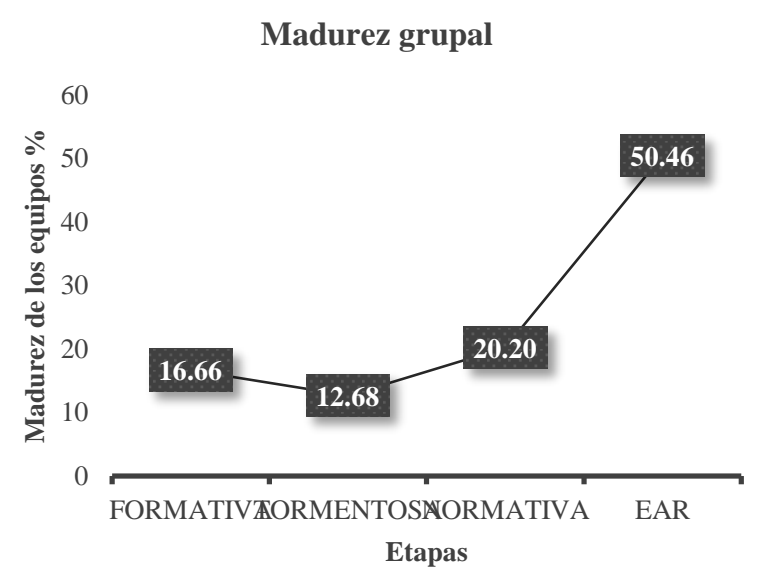

Gráfica 2 Madurez de los EAR de acuerdo a la clasificación de Tuckman

Fuente IPOI-UTH
En la gráfica 2, se muestra la madurez alcanzada por los equipos con un $50.46 \%$ mediante el crecimiento y organización de los integrantes en los equipos durante del desarrollo del proyecto. Los resultados de madurez siguen el patrón de comportamiento de las etapas marcadas en la teoría, del modelo de Tuckman, comentado por Fernández (2012).

\section{Correlación de características de integración de EAR y porcentaje de disminución, a través del pago, en el consumo de energía eléctrica}

Con los resultados anteriores de cohesión y madurez grupal se determinó la correlación entre los porcentajes de cada característica y los obtenidos en la disminución del pago por consumo de energía eléctrica en las casas de los estudiantes que participaron en este proyecto. Los datos se ordenan por pares, cohesión y madurez vs ahorro, respectivamente para cada equipo; se construyeron las gráficas y se determinó el coeficiente de correlación de Pearson para cada grupo.

\section{Correlación de Cohesión grupal VS} Ahorro de energía

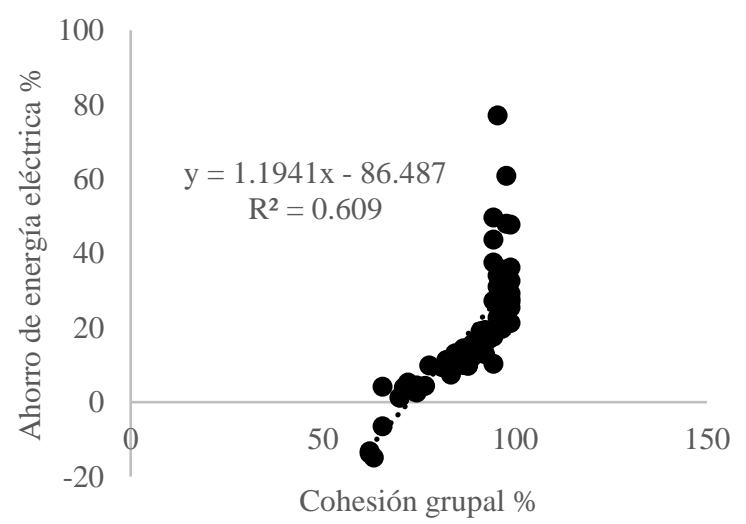

Gráfica 3 Cohesión grupal y su aportación al ahorro de energía eléctrica

Fuente IPOI-UTH

Como resultado de la cohesión grupal alcanzada, en la gráfica 3 se observa un porcentaje predominante alrededor del $90 \%$ lo que contribuye a la disminución del consumo de energía eléctrica, con una concentración entre el 10 y el $40 \%$. Esto, desde nuestro punto de vista, se considera adecuado, pues están respaldados por el coeficiente de correlación de Pearson con un valor de 0.7803 
Con respecto a la madurez alcanzada por los equipos de AR se observa en la gráfica 4 , un impacto en la madurez entre el 70 y el $80 \%$, con una contribución fuerte al ahorro de energía eléctrica, considerando el objetivo inicial del $10 \%$ y con un coeficiente de correlación de Pearson con un valor de 0.8197

\section{Correlación de Madurez grupal VS Ahorro de energía}

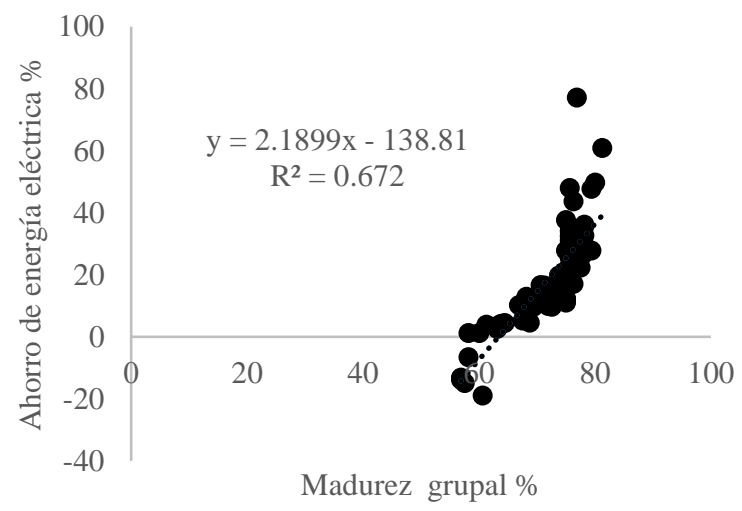

Gráfica 4 Madurez grupal y su aportación al ahorro de energía eléctrica

Fuente IPOI-UTH

\section{Anexos}

Cuestionario de Madurez del Equipo según Bruce Tuckman, Elaborado por Erin Barkema and John W. Moran, octubre 2013 Adaptado en octubre del 2018.

Cuestionaro de cohesión grupal tomado de Dotazník Group Environment Questionnaire (GEQ), Prokešová, Eva, Musálek, Martin, acta universitatis carolinae, kinanthropologica vol. $47,2-2011$

\section{Agradecimiento}

A la UTH por el apoyo para la realización del estudio. A los estudiantes de la generación 20162019, quienes mostraron disponibilidad para el proyecto y aportación de resultados para su análisis estadístico. A los integrantes del CAEF, "gestión de la mentefactura", por sus aportes.

\section{Conclusiones}

Las teorías aplicadas para integrar a los estudiantes de IPOI a EAR, así como los instrumentos utilizados para la evaluación de las actividades indican un comportamiento que cumple con las etapas de conformación de equipos, consiguiendo una cohesión grupal del $54.3 \%$ y una madurez del $50.46 \%$; estos resultados contribuyeron a la reducción de consumo de energía eléctrica para 48 estudiantes de un total de 68 , lo que equivale a un $70.5 \%$ de los participantes en este estudio. El $29.5 \%$ restante corresponde a los estudiantes que por razones de actividades extra clase no se dieron la oportunidad de aprender a trabajar para formar un equipo con características que superen sus resultados a través de la sinergia.

En cuanto a la correlación existente entre las variables de cohesión y madurez grupal contra el ahorro de energía eléctrica, se obtuvieron resultados aceptables para el coeficiente de Pearson con valores de 0.7803 y 0.8197 respectivamente, los cuales dan certeza estadística al estudio. Contribuyendo así, al entendimiento y comprensión de la propuesta del método para conseguir su objetivo de disminuir al menos en un $10 \%$ el pago por el consumo de la energía en sus hogares.

Lo alcanzado hasta aquí, nos da la oportunidad de contribuir en la formación de estudiantes en un esquema que no sigue el patrón tradicional de integración, pues la asociación es innata y conducida para conseguir más de lo que cada uno puede alcanzar de forma individual. A partir de esto, se considera necesaria la implementación en los estudiantes desde su ingreso a la universidad, cultivando la propuesta y enriqueciéndola con aspectos de liderazgo y de inteligencia emocional, con la idea de transitar a un esquema de formación constructivista en asignaturas que por su naturaleza requieran de integración de equipos.

\section{Referencias}

Fernández Alcalde, Josu Bingen (2012), Revista de Ciencias Sociales. Vol. 7, n. ${ }^{\circ}$ 1; pp. 81-108

Herzberg, F., Mausner B., Bloch, B (2010) The motivation to work. Vigésima impresión, Estados unidos de américa. 
IMCO, (2017), Instituto mexicano para la competitividad,

http://imco.org.mx/comparacarreras/

Maslow, Abraham H., (1991) Motivación y personalidad, Ediciones Díaz de Santos, Madrid España.

McGregor, D. M., (1986) «The Human Side of Enterprise», en Yarwood, D. L., Public Administration, Politics and People: Selected Readings for Managers, Employees and Citizens, New York: Longman Publishing Group

Medina, Giacomozzi Alex; Muñoz, Gallegos Cecilia; Hadi, Lara Patricio; (2008) Motivación y satisfacción de los trabajadores y su influencia en la creación de valor económico en la empresa, Revista de Administración Pública, ISSN 00347612, Rio de Janeiro 42(6):1213-30, nov./dez.

Pere, Pujolàs. Lago, José Ramón, (2014) Programa CA/AC para enseñar a aprender en equipo, Universidad de Vic, Cataluña, España

Prokešová, Eva, Musálek, Martin, (2011) Confirmation of conceptual validity of czech version of group environment questionnaire by structural equation modeling, acta universitatis carolinae, kinanthropologica vol. 47, 2 - 2011, Charles university in Prague.

REAL ACADEMIA ESPAÑOLA: Diccionario de la lengua española, 23. a ed., [versión 23.2 en línea]. <https://dle.rae.es> [8/06/2019].

Santrock, John W., (2018) Psicología de la educación 5ta edición, McGraw-Hill, México

"Scoring the Tuckman team maturity questionnaire electronically". https://educationdocbox.com/Homework_and_ Study_Tips/70246021. Consultado en octubre.

Smith, Louis M., (1994) Perspectivas: revista trimestral de educación comparada (París, UNESCO: Oficina Internacional de Educación), vol. XXIV, nos 3-4, págs. 529-542.

Tuckman, B. W. (1965). Psychological Bulletin1965, Vol. 63, No. 6, 384-399, consultado en: https://pdfs.semanticscholar.org/cd78/c763010e 6eb856250b939e4eec438e14ef8f.pdf, mayo 2019.
TLAPALE-HERNÁNDEZ, Prudencio, PÉREZ-RAMOS, María Gabriela, MACUIL-CARMONA, Carolina y MERINO-MUÑOZ, Víctor Hugo. Formación de equipos de alto rendimiento con estudiantes de ingeniería, a través de cohesión y madurez grupal y su aplicación en un proyecto específico. Revista de Ciencias de la Educación. 2019 